\title{
AN ANALOGUE OF THE BAUM-CONNES ISOMORPHISM FOR COACTIONS OF COMPACT GROUPS
}

\author{
RALF MEYER and RYSZARD NEST
}

(Dedicated to the memory of Gert K. Pedersen)

\begin{abstract}
We formulate and prove the analogue of the Baum-Connes conjecture for coactions of connected compact groups.
\end{abstract}

\section{Introduction}

Let $G$ be a second countable locally compact group. Let $A$ be a separable $C^{*}$-algebra with a strongly continuous action of $G$ and let $G \ltimes_{\mathrm{r}} A$ be the reduced crossed product, again a separable $C^{*}$-algebra. The aim of the BaumConnes conjecture (with coefficients) is to compute the K-theory of $G \ltimes_{\mathrm{r}} A$. For $A=C$ endowed with the trivial action of $G$, this becomes a computation of $\mathrm{K}\left(C_{\mathrm{r}}^{*}(G)\right)$, the K-theory of the reduced $C^{*}$-algebra of $G$. One defines a certain graded Abelian group $\mathrm{K}^{\mathrm{top}}(G, A)$, called the topological $\mathrm{K}$-theory of $G$ with coefficients in $A$, and a homomorphism

$$
\mu_{A}: \mathrm{K}^{\mathrm{top}}(G, A) \rightarrow \mathrm{K}\left(A \rtimes_{r} G\right),
$$

which is called the Baum-Connes assembly map. The Baum-Connes conjecture for $G$ with coefficients in $A$ asserts that this map is an isomorphism. It has important applications in topology and ring theory. The conjecture is known to hold in many cases, for instance, for amenable groups ([8]). A recent survey article on the Baum-Connes conjecture is [7].

Our goal is to construct an analogue of topological K-theory and the BaumConnes assembly map for certain discrete quantum groups and their crossed products. In this paper we will implement this program for the first non-trivial case, that of a coaction of a compact group.

Debashish Goswami and A. O. Kuku have proposed such a construction in [5]. Their approach is to extend the usual formulation of the Baum-Connes

Received August 21, 2006. 
assembly map to the quantum group case. We shall follow a different route, the main reason being as follows. The point of the Baum-Connes conjecture for groups is that $\mathrm{K}^{\text {top }}(G, A)$ should be easier to compute than $\mathrm{K}\left(G \ltimes_{\mathrm{r}} A\right)$. This is true, but not obvious from the definition of $\mathrm{K}^{\mathrm{top}}(G, A)$, for the case of locally compact groups. It is not clear at the moment how to compute the topological $\mathrm{K}$-theory for a discrete quantum group as it is defined by Goswami and Kuku.

Our approach to the Baum-Connes assembly map $\mu$ is inspired by an alternative construction of $\mu$ (at least for discrete groups with commutative coefficients) due to James Davis and Wolfgang Lück ([4]). Their construction is purely topological, and the computability of $\mathrm{K}^{\mathrm{top}}(G)$ is more or less built in. As it turns out, there exists a manageable translation of the methods from algebraic topology in [4] into the setting of $C^{*}$-algebras and bivariant KK-theory, which gives a construction of the Baum-Connes assembly map for groups in purely $C^{*}$-algebraic terms. Our approach has two advantages. We do not need to use the classical concept of classifying space, which, in the quantum group case has no natural analogue, and we do not use the concept of a proper group action. Instead, we only need the notion of a proper homogeneous space, which extends in an evident way to quantum groups. Moreover, the construction of the assembly map comes right away with methods to compute $\mathrm{K}^{\mathrm{top}}(G, A)$, also formulated in $C^{*}$-algebraic terms and therefore extend without any change to quantum groups.

The main result of this paper is, loosely stated,

THEOREM. Suppose that $G$ is a compact connected second countable group. Then every element of $\mathrm{KK}^{\hat{G}}$ can be constructed using only proper homogeneous $\hat{G}$-algebras.

The rest of this introduction contains an explanation of this statement.

\subsection{Homological approach in the group case}

Since the basic idea is the extension of the methods and results from [12], we will start by recalling some basics from this paper. Let $G$ be a locally compact, second countable group. $\mathrm{KK}^{G}$ denotes the category, whose objects are separable $C^{*}$-algebras with continuous action of $G$ and whose morphisms are given by the biequivariant $\mathrm{KK}_{0}^{G}$-functors. The composition of morphisms is provided by the Kasparov product

$$
\mathrm{KK}^{G}(A, B) \times \mathrm{KK}^{G}(B, C) \rightarrow \mathrm{KK}^{G}(A, C) .
$$

$\mathrm{KK}^{G}$, while not an abelian category, can be endowed with a triangulated structure, given as follows. 
(1) The shift is given by the (inverse) suspension:

$$
A \rightarrow A[-1]=C_{0}(\mathrm{R}) \otimes_{\min } A
$$

(2) Distinguished triangles are the ones conjugate (in $\mathrm{KK}^{G}$ ) to the triangles associated to cones over $*$-homomorphisms:

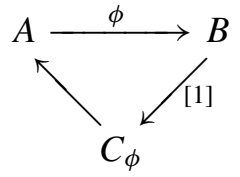

Just as a reminder, let $\phi: A \rightarrow B$ be a $G$-equivariant $*$-homomorphism. Then its mapping cone is given by

$$
\left.\left.C_{\phi}:=\left\{(a, b) \in A \times C_{0}(] 0,1\right], B\right) \mid \phi(a)=b(1)\right\} .
$$

In $\mathrm{KK}^{G}$ any morphism is conjugate to one given by a $*$-homomorphism [11], and the following holds

THEOREM 1.1 (see [12]). $\mathrm{KK}^{G}$ with the above structure is a triangulated category closed under countable direct sums.

For generalities on triangulated categories see [15].

Let $\mathscr{C} C$ denote the full subcategory of $\mathrm{KK}^{G}$ consisting of all objects vanishing in $\mathrm{KK}^{K}$, for all compact subgroups $K$ of $G$. Then one can form the "quotient category"

$$
\mathrm{KK}^{G} / \mathscr{C} C \text {. }
$$

As it turns out, this quotient category is equivalent to the localising subcategory $\mathscr{P}$ of $\mathrm{KK}^{G}$ generated by all proper $G$-algebras. The general theory of triangulated categories produces the machinery of functors localised with respect to $\mathscr{C} C$. To be more precise, given any covariant homological functor from $\mathrm{KK}^{G}$ to the category of say, abelian groups $\mathrm{Ab}$,

$$
F: \mathrm{KK}^{G} \rightarrow \mathrm{Ab},
$$

there exists a left derived functor

$$
\mathrm{L} F: \mathrm{KK}^{G} / \mathscr{C} C \rightarrow \mathrm{Ab}
$$

and a natural transformation

$$
\mu: \mathrm{L} F \Longrightarrow F
$$

such that $F=\mathrm{L} F$ on $\mathscr{P}$. In particular, the following holds. 
TheOREM $1.2([12])$. In the above notation, let $F(A)=K_{*}\left(A \rtimes_{r} G\right)$. Then

$$
K_{\text {top }}^{G}(A)=\mathrm{L} F(A)
$$

and the assembly map coincides with the natural transformation $\mathrm{L} F \Longrightarrow F$.

The general machinery of derived functors provides a "simplicial approximation" (see [2]) for arbitrary objects by objects of $\mathscr{P}$. In our case, all that is involved are $G$-algebras constructed by induction from $H$-algebras, where $H$ varies over the set of compact subgroups of $G$. In particular there exists a universal Dirac-element, i.e. a proper $G$-algebra $P_{C}$ and a morphism $D \in \mathrm{KK}^{G}\left(P_{\mathrm{C}}, \mathrm{C}\right)$, the so called "simplicial approximation" of $\mathrm{C}$, constructed from proper homogeneous actions using standard methods of homological algebra and such that both the derived functors and the assembly map for them can be expressed by

$$
\mathrm{L} F(A)=F\left(P_{\mathrm{C}} \otimes_{\min } A\right) \stackrel{D \otimes 1_{A}}{\longrightarrow} F(A)
$$

In this language various forms of the Baum-Connes conjecture have a very natural, functorial description. Below we will be mainly interested in the following version, which is usually known as the " $\gamma=1$ "-property of $G$.

Strong BAum-Connes CONJECture. The inclusion functor $\mathscr{P} \hookrightarrow \mathrm{KK}^{G}$ is an equivalence of categories.

\subsection{Coaction of compact group case}

We will assume from now on that $G$ is a compact, second countable group. A morphism $\phi: A \rightarrow B$ of not necessarily unital $C^{*}$-algebras is, by definition, given by a $*$-homomorphism from $A$ to the multiplier algebra $\mathcal{M}(B)$ of $B$, satisfying the non-degeneracy condition

$$
\overline{\phi(A) B}=B \text {. }
$$

A morphism of $C^{*}$-algebras extends to a strictly continuous $*$-homomorphism of their multiplier algebras.

$\mathrm{KK}^{\hat{G}}$ denotes the category of $G$-comodules (or, equivalently $\hat{G}$-modules), i.e. separable $C^{*}$-algebras $A$ with a coaction $\Delta$ of $G$, i.e. a morphism

$$
\Delta: A \rightarrow A \otimes_{\min } C^{*}(G)
$$

satisfying two conditions:

- coassociativity: $\left(\Delta \otimes 1_{C^{*}(G)}\right) \circ \Delta=\left(1_{A} \otimes \Delta_{C^{*}(G)}\right) \circ \Delta$, and 
- non-degeneracy: $\Delta(A)\left(1 \otimes C^{*}(G)\right)$ and $\left(1 \otimes C^{*}(G)\right) \Delta(A)$ are both norm dense in $A \otimes_{\min } C^{*}(G)$

Morphisms in $\mathrm{KK}^{\hat{G}}$ are given by elements of equivariant $K K$-theory (see [1]):

$$
\operatorname{Mor}_{\mathrm{KK}^{\hat{G}}}(A, B):=\mathrm{KK}_{0}^{\hat{G}}(A, B) \text {. }
$$

C denotes a $\hat{G}$-algebra with the coaction given by

$$
1 \rightarrow 1 \otimes 1 \in \mathcal{M}\left(\mathrm{C} \otimes_{\min } C^{*}(G)\right)
$$

We endow $\mathrm{KK}^{\hat{G}}$ with a triangulated structure using the equivalence of categories induced by the crossed product functor:

$$
\mathrm{KK}^{G} \ni A \rightarrow A \rtimes G \in \mathrm{KK}^{\hat{G}},
$$

(see [1]) to transfer the triangulated structure of $\mathrm{KK}^{\hat{G}}$ introduced in [12].

\subsection{Quantum homogeneous spaces}

Let us start with a provisoric:

Definition 1.3. Let $\mathscr{H}$ be a quantum group ([10]). The standard notion of a quantum homogeneous $\mathscr{H}$-space is a $*$-coideal in $\mathscr{H}$, i.e. a subalgebra $\mathscr{Q} \subset \mathscr{M}(\mathscr{H})$ such that

$$
\nabla_{\mathscr{H}}: \mathscr{Q} \rightarrow \mathscr{M}\left(\mathscr{H} \otimes_{\min } \mathscr{Q}\right)
$$

( $\mathcal{M}$ stands for multiplier algebra). The classical property of properness for a homogeneous $G$-space translates into:

$$
\mathscr{Q} \subset \mathscr{H}
$$

ExAmple 1.4. As an example, let $\mathscr{H}_{i}, i=1,2$ be quantum groups, $\pi$ : $\mathscr{H}_{1} \rightarrow \mathscr{H}_{2}$ a Hopf $*$-homomorphism. We can define a coaction of $\mathscr{H}_{2}$ on $\mathscr{H}_{1}$ by

$$
\mathscr{H}_{1} \ni x \rightarrow \nabla(x)=\iota \otimes \pi\left(\nabla_{1}(x)\right) \in \mathscr{H}_{1} \otimes_{\min } \mathscr{H}_{2}
$$

The corresponding quantum homogeneous space is

$$
\mathscr{Q}=\left\{x \in \mathscr{H}_{1} \mid \nabla(x)=x \otimes 1\right\},
$$

where the coaction of $\mathscr{H}_{1}$ on $\mathscr{Q}$ is given by:

$$
\nabla_{1}: \mathscr{Q} \rightarrow \mathscr{H}_{1} \otimes_{\min } \mathscr{Q}
$$


This kind of quantum homogeneous space deserves the name classical, with the "stabiliser" $\mathscr{H}_{2}$. However, not every quantum homogeneous space is of this type.

Example 1.5 (Cocommutative quantum group case). A "cocommutative quantum group" is just a classical group. The above just means:

$\mathscr{H}_{1}=C(G)$ and $\mathscr{H}_{2}=C(H) ; \pi(f)=f \circ \phi$, where $\phi: H \rightarrow G$ is a group homomorphism with closed range; $\nabla(f)(g, h)=f(g \phi(h)) ; f \in \mathscr{Q} \Longleftrightarrow$ for all $h \in H f(g \phi(h))=f(g)$, i.e. 2 is $C_{0}(G / \phi(H))$.

EXAMPLE 1.6 (Commutative discrete quantum group). A commutative discrete quantum group $\hat{G}$ is the dual of a compact group $G$. The objects in $\mathrm{KK}^{\hat{G}}$ are $C^{*}$-algebras with a coaction of $G$ and, in the terms of the definition 1.3, $\mathscr{H}=C^{*}(G)$. For the convenience of the reader, let us recall the precise meaning of the "dual" in this case. Let $W$ be the (multiplicative) unitary operator on $\left.L^{2}(G \times G)\right)$ given by

$$
W \xi(g, h)=\xi(g, g h) .
$$

For any $\phi \in B\left(L^{2}(G)\right)_{*}$, we set

$$
a_{\phi}=\left(\phi \otimes \operatorname{id}_{B\left(L^{2}(G)\right)}\right)(W) \quad \text { and } \quad \hat{a}_{\phi}=\mathrm{id}_{B\left(L^{2}(G)\right)} \otimes \phi(W) .
$$

Then $C^{*}(G)$ is the norm closure of the algebra generated by $\left\{a_{\phi} \mid \phi \in\right.$ $\left.B\left(L^{2}(G)\right)_{*}\right\}$ and $C(G)$ is the norm closure of the algebra generated by $\left\{\hat{a}_{\phi} \mid\right.$ $\left.\phi \in B\left(L^{2}(G)\right)_{*}\right\}$, both in $B\left(L^{2}(G)\right)$. The bilinear form

$$
B\left(a_{\phi}, \hat{a}_{\psi}\right)=(\phi \otimes \psi)(W)
$$

defines a non-degenerate pairing between dense subsets of $C^{*}(G)$ and $C(G)$.

In more concrete terms, let $\lambda: C(G) \rightarrow C^{*}(G)$ be the integrated left regular representation of $G$ and $L=\mathrm{id}: C(G) \rightarrow C(G)$ be the integrated left regular representation of $\hat{G}$. Then the pairing above reduces to

$$
B(\lambda(f), L(g))=\int_{G} f g d \mu,
$$

where $d \mu$ is the Haar measure on $G$. Note that $B$ does not extend to all of $C^{*}(G) \times C(G)$.

Let $C_{\text {alg }}(G)$ denote the subalgebra of $C(G)$ generated by the matrix coefficients of irreducible subrepresentations of the left regular representation. The Peter-Weyl theory implies the following facts. 
- $\lambda\left(C_{\mathrm{alg}}(G)\right)$ is dense in $C^{*}(G)$ and consists of finite rank operators, in fact, $C^{*}(G)$ is a ( $C^{*}$-algebraic) direct sum of a countable family of finitedimensional matrix algebras,

$$
C^{*}(G)=\hat{\oplus}_{\pi} M_{d_{\pi}}(\mathrm{C}), \quad \pi \text { irreducible representation of } G
$$

and $C_{\text {alg }}(G)$ is the subalgebra given by the algebraic direct sum

$$
\oplus_{\pi}^{\mathrm{alg}} M_{d_{\pi}}(\mathrm{C})
$$

- for any $C^{*}$-co-ideal $\mathscr{Q}$ in $C^{*}(G), \mathscr{Q}_{\text {alg }}=\mathscr{Q} \cap \lambda\left(C_{\text {alg }}(G)\right)$ is a dense $*$ subalgebra of 2 ;

- for any $C^{*}$-subalgebra $\mathscr{Q}$ of $C^{*}(G), \mathscr{Q}_{\text {alg }}$ is a direct sum of matrix algebras and

$$
\Delta_{C^{*}(G)}\left(\mathscr{Q}_{\text {alg }}\right) \subset \mathscr{Q}_{\text {alg }} \otimes_{\text {alg }} C^{*}(G)_{\text {alg }} .
$$

Suppose that $\mathscr{Q}$ is a (left, norm closed) coideal in $C^{*}(G)$. Then the annihilator of $\mathscr{Q}_{\text {alg }}$,

$$
I=\left\{f \in C(G) \mid B(X, L(f))=0 \text { for all } X \in \mathscr{Q}_{\text {alg }}\right\}
$$

is a norm closed ideal in $C(G)$ and hence there exists an open subset $H$ of $G$ such that $I=\left\{f \in C(G)|f|_{G \backslash H}=0\right\}$. In other words, for $f \in C(G)$,

$$
\lambda(f) \in \mathscr{Q} \Longleftrightarrow \operatorname{supp}(f) \subset H .
$$

Since 2 is a $*$-subalgebra of $C^{*}(G), H$ has to be an open subgroup of $G$ and $2=C^{*}(H)$ as a subalgebra of $C^{*}(G)$. All together, we obtained a bijection:

proper homogeneous subspaces of $C^{*}(G) \longleftrightarrow$ open subgroups of $G$,

Note that there is no analogue of $\mathscr{H}_{2}$ (the stabiliser subgroup) in this case, unless the open subgroup in question is normal.

In the case of actions of locally compact groups $G$ the class of proper homogeneous spaces already generates all proper actions of $G$. As it turns out, this is not quite enough, even for our simplest example of a quantum group which is the dual of a compact group. Before giving the definition, we will need a bit more notation.

Notation 1.7. For any locally compact group $K$ and any two-cocycle $\omega \in H^{2}(K, T)$,we let $\left(\lambda_{\omega}, L^{2}(K)\right)$ denote the projective unitary representation of $K$ on $\left.L^{2}(K)\right)$ given by

$$
\left(\lambda_{\omega}(k) f\right)(l)=\omega(k, l) f\left(k^{-1} l\right) .
$$


Given such a pair $(K, \omega), \mathrm{C}_{\omega}$ will denote the $C^{*}$-algebra of compact operators on $L^{2}(K)$ with the $\operatorname{Ad} \lambda_{\omega}$ action of $K$.

Given a compact, separable group $G$, we denote by $\mathscr{I}_{G}$ the family of $G$ coalgebras of the form

$$
\left(\operatorname{Ind}_{K_{0}}^{K} \mathrm{C}_{\omega}\right) \rtimes G
$$

where:

(i) $K$ is a Lie-group quotient of $G, K_{0}$ its component of identity and $\omega \in$ $H^{2}\left(K_{0}, T\right)$ a finite valued cocycle;

(ii) $\operatorname{Ind}_{K_{0}}^{K} \mathrm{C}_{\omega}$ is the induced $K-C^{*}$-algebra with the action of $G$ induced by the homomorphism $G \rightarrow K$.

DEFINITION 1.8. A $G$-coalgebra is called a properhomogeneous $\hat{G}$-algebra if it belongs to $\mathscr{I}_{G}$;

A $G$-coalgebra $A \in \mathrm{KK}^{\hat{G}}$ is called a cofibrant $\hat{G}$-algebra if it belongs to the localising subcategory of $\mathrm{KK}^{\hat{G}}$ generated by the elements of the form $Y \otimes_{\min } P$ with $Y$ a trivial $\hat{G}$-algebra and $P$ a proper homogeneous $\hat{G}$-algebra. We will denote this subcategory by $\mathscr{P}$.

REMARK 1.9. In the case of connected compact groups, the above amounts to defining proper homogeneous $\hat{G}$-algebras to be of the form $C^{*}(G, \omega)$, with $\omega$ a finite-valued two cocycle on the group. Even if $\hat{G}$ has no "classical torsion", it still may have "quantum torsion", coming from twisting of the product by torsion 2-cocycles. The term "cofibrant" instead of proper as used in the group case, comes from homological properties of the subcategory $\mathscr{P}$.

\subsection{Baum-Connes isomorphism for duals of compact groups}

As usual, Baum-Connes isomorphism say nothing in the case of duals of finite groups. This makes the case of the connected compact groups more transparent, and we will deal mainly with this case. The precise statements, proved in the last section of this paper, are as follows (see Theorems 3.6).

THEOREM. Let $G$ be a compact connected group. Every object in $\mathrm{KK}^{\hat{G}}$ is cofibrant.

As an example of an application, let us note the following:

COROLlary. Let $G$ be a connected second countable compact group and $B$ a separable $C^{*}$-algebra with action of $G$. Suppose that $G$ has a sequence of subgroups $H_{k}$ such that $\cap_{k} H_{k}=\{e\}$ and $G / H_{k}$ is a Lie group with torsionfree fundamental group. Then, if $K_{*}\left(B^{H_{k}} \rtimes\left(G / H_{k}\right)\right)=0$ for all $k$, then the $K$-theory of $B$ vanishes. 
For the complete statement and the proof see the corollary 3.8. The particular case of connected simply connected Lie groups has been studied in [17].

\section{The case of $C$ and $C(G / T) \rtimes G$}

Let us recall that, given a triangulated category $\mathscr{C}$ and a family $\mathscr{T}$ of objects of $\mathscr{C}$, the localising subcategory of $\mathscr{C}$ generated by $\mathscr{T}$ is the smallest subcategory of $\mathscr{C}$ containing $\mathscr{T}$ and closed under the shift, the mapping cone construction and retracts.

Proposition 2.1. Suppose that $G$ is a compact connected Lie group and $T$ its maximal torus. Then the $G$-module $C(G / T)$ belongs to the localising subcategory of $\mathrm{KK}^{G}$ generated by the G-modules of the form $\mathrm{C}_{\omega}$, where $\omega$ is a torsion class (i.e. has a finite-valued representative) in $H^{2}\left(G, S^{1}\right)$.

Proof. The proof will be done in two steps.

2.0.1. Hodgkins groups. Given a compact group $G$, we will use $R_{G}$ to denote its representation ring which is isomorphic to $\mathrm{KK}^{G}(\mathrm{C}, \mathrm{C})$. For any $A$ and $B$ in $\mathrm{KK}^{G}, \mathrm{KK}^{G}(A, B)$ has a natural left $R_{G}$-module structure given by the Kasparov exterior tensor product:

$$
\mathrm{KK}^{G}(\mathrm{C}, \mathrm{C}) \times \mathrm{KK}^{G}(A, B) \rightarrow \mathrm{KK}^{G}(A \otimes \mathrm{C}, B \otimes \mathrm{C})=\mathrm{KK}^{G}(A, B) .
$$

Lemma 2.2. Let $G$ be a compact Lie group and let $T$ denote its maximal torus. Suppose that $\pi_{1}(G)$ is torsion free (i.e. $G$ is a Hodgkins group). Let $\mathscr{S}$ denote the localising subcategory of $\mathrm{KK}^{G}$ generated by $C(G / T)$ and $\mathrm{C}$. Given any $A \in \operatorname{Obj}(\mathscr{S})$, the natural homomorphism of abelian groups

$$
j_{A}: R_{T} \otimes_{R_{G}} \mathrm{KK}^{G}(A, \mathrm{C}) \rightarrow \mathrm{KK}^{T}(A, \mathrm{C})
$$

given by the restriction of the second factor to $\mathrm{KK}^{T}$ followed by multiplication is an isomorphism.

Proof. Since the map is given by a natural transformation between two cohomological functors, it is enough to check the claim on the generators of $\mathscr{S}$.

(i) For $A=C$. Since the trivial representation of $G$ acts as a unit on $R_{T}$,

$$
R_{T} \otimes_{R_{G}} \mathrm{KK}^{G}(\mathrm{C}, \mathrm{C})=R_{T} \otimes_{R_{G}} R_{G} \stackrel{j \mathrm{c}}{\longrightarrow} R_{T}
$$

is an isomorphism.

(ii) For $A=C(G / T)$. Since $G$ is connected and $\pi_{1}(G)$ is torsion free, the following are true.

- $R_{T} \otimes_{R_{G}} R_{T}=K(C(G / T) \rtimes T)($ see McLeod [13]); 
- Poincare duality:

$$
\mathrm{KK}^{G}(C(G / T), \mathrm{C}) \simeq \mathrm{KK}^{G}(\mathrm{C}, C(G / T))
$$

as $R_{G}$-modules ([17]);

- Green-Julg theorem ([6]): $K(A \rtimes T)=\mathrm{KK}^{T}(\mathrm{C}, A)$;

- induction-restriction adjunction in $\mathrm{KK}^{G}$ - given a $G$-algebra $A$ and a $T$ algebra $B$, the following holds:

$$
\mathrm{KK}^{T}(A, B)=\mathrm{KK}^{G}\left(A, \operatorname{Ind}_{T}^{G}(B)\right)
$$

(see [17]).

The claim follows by noting that $j_{C(G / T)}$ is given by the string of isomorphisms:

$$
\begin{aligned}
R_{T} \otimes_{R_{G}} \mathrm{KK}^{G}(C(G / T), \mathrm{C}) & \simeq R_{T} \otimes_{R_{G}} R_{T} \simeq K(C(G / T) \rtimes T) \\
& \simeq \mathrm{KK}^{T}(\mathrm{C}, \mathrm{C}(G / T)) \simeq \mathrm{KK}^{T}(\mathrm{C}(G / T), \mathrm{C}) .
\end{aligned}
$$

2.0.2. Proof of Proposition 2.1. Suppose first that $\pi_{1}(G)$ is torsion free. Then (by [18]), $R_{T}$ is finitely generated and free over $R_{G}$. Let $\mathrm{n}$ denote its rank. The above lemma shows that the two representable functors on $\mathscr{S}$ :

$$
A \Longrightarrow \mathrm{KK}^{G}(A, C(G / T))=\mathrm{KK}^{T}(A, \mathrm{C})
$$

and

$$
A \Longrightarrow R_{T} \otimes_{R_{G}} \mathrm{KK}^{G}(A, \mathrm{C}) \simeq \mathrm{KK}^{G}\left(A, \mathrm{C}^{n}\right)
$$

coincide. By the Yoneda lemma this implies that

$$
C(G / T)=\mathrm{C}^{n} \text { in } \mathscr{S} \text { and hence in } \mathrm{KK}^{G} .
$$

Let now $G$ be an arbitrary connected compact Lie group. Let $\tilde{G}$ be a finite connected covering group of $G$ with a finite central subgroup $Z$ such that $\pi_{1}(\tilde{G})$ is torsion free and $G=\tilde{G} / Z$. By the above,

$$
C(\tilde{G} / \tilde{T}) \in\langle\mathrm{C}\rangle
$$

holds in $\mathrm{KK}^{\tilde{G}}$.

Let us recall the partial descent functor [3]

$$
F_{\rtimes Z}: \mathrm{KK}^{\tilde{G}} \ni A \rightarrow A \rtimes Z \in \mathrm{KK}^{G}
$$

constructed as follows. Given a $\tilde{G}$ - $C^{*}$-algebra $A$, the crossed product $A \rtimes \tilde{G}$ admits a coaction $\Delta$ of $\tilde{G}$ given by $\operatorname{id}_{A} \otimes \Delta_{\tilde{G}}$ and hence the coaction of $G$ given 
by $\left(\operatorname{id}_{A} \otimes q\right) \circ\left(\operatorname{id}_{A} \otimes \Delta_{\tilde{G}}\right)$, where $q: \tilde{G} \rightarrow G$ denotes the quotient map. The double crossed product

$$
(A \rtimes \tilde{G}) \rtimes \hat{G}
$$

has a natural (dual) action of $G$ and represents $F_{\rtimes Z}(A)$ in $\mathrm{KK}^{G}$. By construction $F_{\rtimes Z}$ commutes both with the suspension and the mapping cone construction, hence is triangulated and the equation (4) implies that

$$
F_{\rtimes Z}(C(\tilde{G} / \tilde{T})) \in\left\langle F_{\rtimes Z}(\mathrm{C})\right\rangle .
$$

It is easy to see that $F_{\rtimes Z}(C(\tilde{G} / \tilde{T}))$ splits as a direct sum of $G$-modules of the form $\mathrm{C}_{\omega} \otimes_{\min } C(G / T)$, where $\omega$ runs over all two-cocycles of $G$ induced by the characters of $Z$. In particular, one of the summands is $C(G / T)$. Since $F_{\rtimes Z}(\mathrm{C})$ is given by the direct sum of $G$-modules of the form $\mathrm{C}_{\omega}$, the claimed result follows.

The following corollary of the proof will be useful later.

Corollary 2.3. Suppose that $G$ is a compact connected Lie group and $T$ its maximal torus. Then the $G$-module $\oplus C_{\eta} \otimes C(G / T)$ contains a $G$-module of the form $\mathrm{C}_{\omega}$ as a direct summand.

COROLlaRY 2.4. Suppose that $G$ is a compact connected Lie group and $T$ its maximal torus. Then the $G$-module $C(G)$ belongs to the localising subcategory of $\mathrm{KK}^{G}$ generated by the $G$-modules of the form $\mathrm{C}_{\omega}$, where $\omega$ is a torsion class (i.e. has a finite-valued representative) in $H^{2}\left(G, \mathrm{~T}^{1}\right)$.

Proof. Let $T$ be a maximal torus in $G$. Then

$$
C(G) \in\langle C(G / T)\rangle \quad \text { in } \mathrm{KK}^{G} .
$$

In fact, since $\hat{T}=\mathrm{Z}^{r}$ is discrete abelian and torsion free, the strong Baum Connes conjecture holds for $\hat{T}$, which in our language means that

$$
\mathrm{C} \in\left\langle c_{0}(\hat{T})\right\rangle \quad \text { in } \mathrm{KK}^{\hat{T}} .
$$

By the (Takesaki-Takai) duality between $\mathrm{KK}^{T}$ and $\mathrm{KK}^{\hat{T}}$,

$$
C(T)=C^{*}(\hat{T}) \in\langle\mathrm{C}\rangle \quad \text { in } \mathrm{KK}^{T} .
$$

Since the induction

$$
\mathrm{KK}^{T} \ni A \rightsquigarrow \operatorname{Ind}_{T}^{G}(A) \in \mathrm{KK}^{G}
$$

is a triangulated functor, this implies that

$$
C(G)=\operatorname{Ind}_{T}^{G} C(T) \in\left\langle\operatorname{Ind}_{T}^{G} \mathrm{C}\right\rangle=\langle C(G / T)\rangle .
$$

Together with the proposition 2.1 this implies the claim of the corollary. 


\section{Non-trivial coefficients}

\subsection{The case of compact connected Lie groups}

Let $G$ be a compact connected Lie group and let $T$ denote the maximal torus of $G$. We will use the notation

$$
A \Longrightarrow \mathrm{t}(A)
$$

for the forgetful functor

$$
\mathrm{KK}^{G} \Longrightarrow \mathrm{KK} .
$$

Lemma 3.1. For any separable G-algebra $A$

$$
A \in\left\langle\operatorname{Ind}_{T}^{G}\left(A \otimes_{\min } \mathrm{C}_{\omega}\right), \omega \in H^{2}\left(G, \mathrm{~T}^{1}\right) \text { finite-valued }\right\rangle
$$

holds in $\mathrm{KK}^{G}$.

Proof. Since $A$ is a $G$-algebra, $\operatorname{Ind}_{T}^{G} A \simeq A \otimes C(G / T)$ and an application of the corollary 2.3 implies the claimed result.

Lemma 3.2. For any separable G-algebra A,

$$
A \in\langle\mathrm{t}(A \rtimes T)\rangle
$$

holds in $\mathrm{KK}^{T}$.

Proof. In fact, since the strong Baum-Connes conjecture holds for the free abelian group $\hat{T}$,

$$
\mathrm{C} \in\left\langle c_{0}(\hat{T})\right\rangle \quad \text { in } \mathrm{KK}^{\hat{T}}
$$

hence, taking crossed product with $\hat{T}$, we get

$$
\begin{aligned}
A \simeq(A \rtimes T) \rtimes \hat{T} \in & \left\langle\left((A \rtimes T) \otimes_{\min } c_{0}(\hat{T})\right) \rtimes \hat{T}\right\rangle \\
& =\left\langle(\mathrm{t}(A \rtimes T)) \otimes_{\min }\left(c_{0}(\hat{T}) \rtimes \hat{T}\right)\right\rangle=\langle\mathrm{t}(A \rtimes T)\rangle
\end{aligned}
$$

as claimed.

Proposition 3.3. Suppose that $G$ is a compact, connected Lie group and $A$ is a separable G-algebra. Then

$$
A \in\left\langle\mathrm{t}\left(\left(A \otimes_{\min } \mathrm{C}_{\mu}\right) \rtimes T\right) \otimes_{\min } \mathrm{C}_{\omega}, \omega, \mu \in H^{2}\left(G, \mathrm{~T}^{1}\right) \text { torsion }\right\rangle
$$

holds in $\mathrm{KK}^{G}$.

Proof. Using the above two lemmas we get, in $\mathrm{KK}^{G}$,

$$
\begin{aligned}
A \in\left\langle\operatorname{Ind}_{T}^{G}\left(A \otimes_{\min } \mathrm{C}_{\mu}\right)\right\rangle \subset\left\langle\operatorname{Ind}_{T}^{G}\left(\mathrm{t}\left(A \otimes_{\text {min }} \mathrm{C}_{\mu}\right) \rtimes T\right)\right\rangle \\
\quad=\left\langle\mathrm{t}\left(\left(A \otimes_{\text {min }} \mathrm{C}_{\mu}\right) \rtimes T\right) \otimes_{\text {min }} C(G / T)\right\rangle
\end{aligned}
$$


but, by Proposition 2.1,

$$
\begin{aligned}
\left\langle\mathrm{t}\left(\left(A \otimes_{\min } \mathrm{C}_{\mu}\right) \rtimes T\right)\right. & \left.\otimes_{\min } C(G / T)\right\rangle \\
& \subset\left\langle\mathrm{t}\left(\left(A \otimes_{\min } \mathrm{C}_{\mu}\right) \rtimes T\right) \otimes_{\min } \mathrm{C}_{\omega}, \omega, \mu \in H^{2}(G, T)\right\rangle,
\end{aligned}
$$

again in $\mathrm{KK}^{G}$.

Corollary 3.4. Let $G$ be a compact, connected Lie group, $T$ its maximal torus, and $B$ a separable $G$-coalgebra. Set

$$
\mathscr{T}(B)=\left\{\begin{array}{l|l}
X & \begin{array}{l}
X \text { is a trivial } G \text {-comodule of the form } \\
\left.(B \rtimes \hat{G}) \otimes_{\min } C_{\omega}\right) \rtimes T, \omega \in H^{2}\left(G, \mathrm{~T}^{1}\right) \text { torsion }
\end{array}
\end{array} .\right.
$$

Then, in $\mathrm{KK}^{\hat{G}}$,

$$
\left.B \in\left\langle X \otimes_{\min } C^{*}(G, \omega)\right| X \in \mathscr{T}(B), \omega \in H^{2}(G, T) \text { torsion }\right\rangle .
$$

Proof. The claim follows immediately from above by writing $B$ (up to stable isomorphism) in the form $A \rtimes G$ for a $G-C^{*}$-algebra $A$ and by applying the Baaj-Skandalis duality ([1]) from $\mathrm{KK}^{G}$ to $\mathrm{KK}^{\hat{G}}$.

\subsection{The general connected compact group case}

THEOREM 3.5. Let $G$ be a compact connected group. Every separable $G-C^{*}$ algebra is $\mathrm{KK}^{G}$-equivalent to an object in the localising subcategory of $\mathrm{KK}^{G}$ generated by $G$ - $C^{*}$-algebras of the form

$$
Y \otimes_{\min } \mathrm{C}_{\omega}
$$

where

- $K$ is a compact Lie group which is a quotient of $G, \omega$ is a finite valued two-cocycle on $K$ and

- $Y$ is a separable $C^{*}$-algebra with trivial action of $G$.

Moreover, given a concrete $A$ in $\mathrm{KK}^{G}$, the involved $Y$ 's are all of the form $\left(A^{H} \otimes_{\min } \mathrm{C}_{\mu}\right) \rtimes T$, where $H$ is the kernel of the quotient homomorphism $G \rightarrow K, T$ is the maximal torus of $K$ and $\mu$ is a finite valued two-cocycle on $K$.

Proof. Let $G$ be a connected compact group and $A$ a separable $G$-algebra. By the structure theory of second countable compact groups (cf. [14]), $G$ can be written as a projective limit

$$
G=\lim _{\longleftarrow}\left\{G^{n}\right\}
$$


of a sequence of compact connected Lie groups $G^{n}$. Let $\pi_{n}: G \rightarrow G^{n}$ denote the induced homomorphisms. We can, and will, assume that $\pi_{n}$ are surjective. Then $\pi_{n}$ induce functors

$$
\mathrm{KK}^{G^{n}} \rightsquigarrow \mathrm{KK}^{G} .
$$

Let $H^{n}$ be the kernel of $\pi_{n}: G \rightarrow G^{n}$. Set $A_{n}=A^{H^{n}}$. Then $A_{n}$ is a separable $G^{n}$-algebra hence, by the proposition 3.3, $A_{n}$, as $G^{n}$ - and hence $G$-module, belongs to the localising category generated by the objects of the form $Y \otimes_{\min } \mathrm{C}_{\omega}$ as described in the theorem. Moreover

$$
A=\underset{\lim }{\longrightarrow}\left\{A_{n}\right\} .
$$

In fact, let $d \mu_{n}$ denote the normalised Haar measure on $H_{n}$. Since $G$ is compact, for any $a \in A$,

$$
\lim _{n \rightarrow \infty} \sup _{h \in H_{n}}\|h(a)-a\|=0,
$$

hence the projections

$$
\Pi_{n}: A \ni a \rightarrow \int_{H_{n}} h(a) d \mu_{n}(h) \in A_{n}
$$

satisfy $\Pi_{n}(a) \rightarrow a$ as $n \rightarrow \infty$. Since localising subcategories are closed under admissible countable direct limits [12], this implies that

$$
A \in\left\langle\bigcup_{n}\left\{Y \otimes_{\min } \mathrm{C}_{\omega}, Y \text { trivial } G^{n} \text {-module }\right\}\right\rangle .
$$

THEOREM 3.6. Suppose that $G$ is a compact connected group. Then every element of $\mathrm{KK}^{\hat{G}}$ is a cofibrant $\hat{G}$-algebra.

More precisely, let $H_{k}$ be any sequence of closed normal subgroups of $G$ such that $\cap_{k} H_{k}=\{e\}$ and $G / H_{k}$ is a Lie group with the maximal torus $T_{k}$. Any $A \in \mathrm{KK}^{\hat{G}}$ is $\mathrm{KK}^{\hat{G}}$-equivalent to a $C^{*}$-algebra constructed (using mapping cones, suspension, direct limits and retracts) from $\hat{G}$-algebras of the form

$$
Y \otimes_{\min } P
$$

with $P$ a proper homogeneous $\hat{G}$-algebra and $Y$ a $C^{*}$-algebra of the form

$$
\left((A \rtimes \hat{G})^{H_{k}} \otimes_{\min } C_{\omega}\right) \rtimes T_{k},
$$

with trivial $\hat{G}$ action.

PRoof. This follows immediately by applying the crossed product functor $A \rightsquigarrow A \rtimes G$ to the theorem 3.5. 
Lemma 3.7. Suppose that $G$ is a connected Lie group with maximal torus $T$, and $B$ is a separable $C^{*}$-algebra with continuous action of $G$. Then

$$
\left\{\begin{array}{l}
K_{*}\left(\left(B \otimes_{\min } C_{\omega}\right) \rtimes G\right)=0 \\
\text { for all finite-valued } \omega \in H^{2}\left(G, \mathrm{~T}^{1}\right)
\end{array}\right\} \Longrightarrow\left\{K_{*}(B \rtimes T)=0\right\}
$$

Proof. First of all, $B \rtimes T$ is Morita equivalent to $\left(B \otimes_{\min } C(G / T)\right) \rtimes G$. Applying theorem 3.6 above to the trivial $\hat{G}$ algebra $C$, we can conclude that, in $\mathrm{KK}^{G}, C(G / T)$ is constructed from $\mathrm{C}_{\omega}$ 's. In particular,' the $K$-theory of $B \rtimes T$ is constructed from the $K$-theory of $\left(B \otimes_{\min } C_{\omega}\right) \rtimes G$. This implies the claim of the lemma.

Corollary 3.8. Let $G$ be a connected compact group and $B$ a separable $C^{*}$-algebra with an action of $G$. Suppose that $G$ has a sequence of subgroups $H_{k}$ such that

$$
\cap_{k} H_{k}=\{e\} \text { and } G / H_{k}
$$

is a Lie group. Suppose moreover that, for all $k$,

$$
K_{*}\left(\left(B^{H_{k}} \otimes \mathrm{C}_{\omega}\right) \rtimes\left(G / H_{k}\right)\right)=0
$$

for all finite valued $\omega \in H^{2}\left(G / H_{k}, \mathrm{~T}^{1}\right)$. Then

$$
K_{*}(B)=0 .
$$

In particular, if $G$ is a connected Lie group and $\pi_{1}(G)$ has no torsion, then vanishing of the $K$-groups of $B \rtimes G$ implies vanishing of the $K$-groups of $B$ (see [16]).

Proof. Let $T_{k}$ denote the maximal torus of $G / H_{k}$. Our assumptions together with the above lemma imply that, for all $k$,

$$
K_{*}\left(B^{H_{k}} \rtimes T_{k}\right)=0 .
$$

But then, by the theorem 3.5 and the Künneth formula, $B$ belongs to the localising subcategory of KK generated by a collection of elements with vanishing $K$-groups and hence the $K$-groups of $B$ have to vanish as well.

\section{REFERENCES}

1. Baaj, Saad, and Skandalis, Georges, $C^{*}$-algèbres de Hopf et théorie de Kasparovéquivariante, $K$-Theory 2 (1989), no. 6, 683-721.

2. Christensen, J. Daniel, Ideals in triangulated categories: phantoms, ghosts and skeleta, Adv. Math. 136 (1998), no. 2, 284-339. 
3. Chabert, Jérôme, and Echterhoff, Siegfried, Twisted equivariant KK-theory and the BaumConnes conjecture for group extensions, $K$-Theory 23 (2001), no. 2, 157-200.

4. Davis, James F., and Lück, Wolfgang, Spaces over a category and assembly maps in isomorphism conjectures in $K$ - and $L$-theory, $K$-Theory 15 (1998), no. 3, 201-252.

5. Goswami, Debashish, and Kuku, A. O., A complete formulation of the Baum-Connes conjecture for the action of discrete quantum groups, Special issue in honor of Hyman Bass on his seventieth birthday. Part IV, $K$-Theory 30 (2003), no. 4, 341-363.

6. Julg, Pierre, $K$-théorie équivariante et produits croisés, C. R. Acad. Sci. Paris Sér. I Math. 292 (1981), no. 13, 629-632.

7. Higson, Nigel, The Baum-Connes conjecture, Proceedings of the international congress of mathematicians II (Berlin, 1998), 1998, 637-646 (electronic).

8. Higson, Nigel and Kasparov, Gennadi, E-theory and KK-theory for groups which act properly and isometrically on Hilbert space, Invent. Math. 144 (2001), no. 1, 23-74.

9. Kasparov, G. G., Equivariant KK-theory and the Novikov conjecture, Invent. Math. 91 (1988), no. 1, 147-201.

10. Kustermans, J., and Vaes, S., Locally compact quantum groups, Ann. Sci. École Norm. Sup. 33 (2000), no. 6, 837-934.

11. Meyer, Ralf, Equivariant Kasparov theory and generalized homomorphisms, $K$-Theory 21 (2000), no. 3, 201-228.

12. Meyer, Ralf, and Nest, Ryszard, The Baum-Connes conjecture via localization of categories, Topology (2006).

13. McLeod, John, The Künneth formula in equivariant $K$-theory, in Algebraic Topology, Waterloo, 1978 (Proc. Conf., Univ. Waterloo, Waterloo, Ont., 1978), Lecture Notes in Math. 741 (1979), 316-333.

14. Montgomery, D., and Zippin, L., Topological Transformation Groups, Interscience, 1955.

15. Neeman, Amnon, Triangulated Categories, Ann. of Math. Stud. 148 (2001).

16. Phillips, N. Christopher, Crossed products of the Cantor set by free minimal actions of $\mathbf{Z}^{d}$, Comm. Math. Phys. 256 (2005), no. 1, 1-42.

17. Rosenberg, J., and Schochet, C., The Künneth formula and the universal coefficient theorem in equivariant $K$-theory and $K K$-theory, Mem. Amer. Math. Soc. 348.

18. Steinberg, Robert, On a theorem of Pittie, Topology 14 (1975), 173-177.

19. Verdier, Jean-Louis, Des catégories dérivées des catégories abéliennes, Astérisque 239 (1996).

MATHEMATISCHES INSTITUT

GEORG-AUGUST-UNIVERSITÄT GÖTTINGEN

BUNSENSTR. 3-5

37073 GÖTTINGEN

GERMANY

E-mail: meyerr@member.ams.org
INSTITUT FOR MATEMATISKE FAG KØBENHAVNS UNIVERSITET UNIVERSITETSPARKEN 5 2100 KØBENHAVN DENMARK

E-mail: rnest@math.ku.dk 